\title{
DETECTING BRAIN CONNECTIVITY CHANGES DURING SEIZURES USING GRANGER CAUSALITY MEASURES ON SCALP EEG
}

\author{
Christos Koutlis ${ }^{1}$, Pål G. Larsson ${ }^{2}$ and Dimitris Kugiumtzis ${ }^{1}$ \\ ${ }^{1}$ Department of Electrical and Computer Engineering, Aristotle University of Thessaloniki, Thessaloniki 54124, Greece, ${ }^{2}$ Department of Neurology, Oslo University Hospital, Norway
}

\section{Objective}

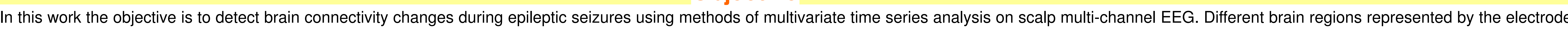

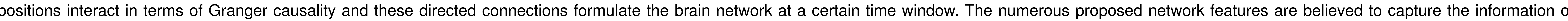

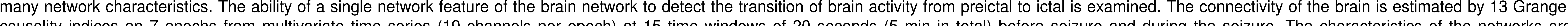

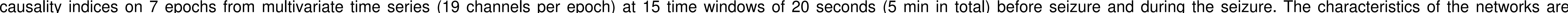
estimated by 379 network features. Finally, the discrimination task (preictal vs. ictal) for each network feature is evaluated by the area under receiver operating characteristic curve (AUROC).

\section{A. Electroencephalograms - EEG Data}

(provided by the Division of Neuroscience, Rikshospitalet, Oslo, Norway)

7 preictal scalp EEG records:

Single seizure episode of 7 patients.

All (but one) are generalized tonic clonic (GTC) seizures.

At least $3 \mathrm{~h}$ and $10 \mathrm{~min}$ duration prior to seizure onset.

19 channels (frontal channels excluded), 10-20 system.

Sampling time $0.01 \mathrm{~s}$ (after subsampling),

Filtering (high-pass at $0.3 \mathrm{~Hz}$, low-pass at $40 \mathrm{~Hz}$ )

No other data pre-processing.

Consecutive non-overlapping segments of duration 20 s (time series length $=2000$ )

Classes

From each EEG record two brain states were used for the discrimination task: Pre-ictal: the last $5 \mathrm{~min}$ before the seizure onset, totally 15 segments of $20 \mathrm{~s}$ Ictal: from $1 \mathrm{~min} 10 \mathrm{~s}$ to $13 \mathrm{~min} 44 \mathrm{~s}$ depending on the duration of the seizure.

B. Causality measures and Network Features

B1. Five Granger causality measures $[1,2]$

Representative Granger causality (GC) measures, selected to be computationally efficient, from time (1) frequency (2) state (3) and phase (4) domain are used:

(1). Restricted conditional Granger causality index RCGCl

(2a). Restricted Generalized Partial Directed Coherence at the bands $\delta, \theta$, a $\beta, y$, denoted RGPDCd, RGPDCt, RGPDCa, RGPDCb and RGPDCg. (2b). Imaginary Coherence at the bands $\delta, \theta, \alpha, \beta, \gamma$, denoted iCOHd, iCOHt, $\mathrm{iCOHa}$, iCOHb and $\mathrm{iCOHg}$

(3). Synchronization likelihood (SL)

(4). Weighted phase lag index (WPLI).

Measures (1) and (2a) are the corresponding to CGCl and GPDC making use of dimension reduction (selecting a subset of terms in VAR model).

A weighted causality network is formed from each GC(i->i) measure computed for all (i,j) pairs at each segment.

B2. Network Features [3]

A large number (379) of network measures were computed at each causality network, including:

- global network measures (mean, std, skewness, kurtosis of the strength

distribution, radius, diameter, centrality, transitivity ratio, eccentricity, clustering

coefficient etc.) and

- the respective local (node-specific) measures.

\section{Methodology}

C. Evaluation of network measures with AUROC [4]

The ability of the network features to discriminate ictal from pre-ictal activity is assessed by the area under receiver operating characteristic curve (AUROC). If AUROC $=1$ there is perfect discrimination of the two classes (the feature values in the two groups do not overlap), if $A U R O C=0.5$ the classification is considered as random.

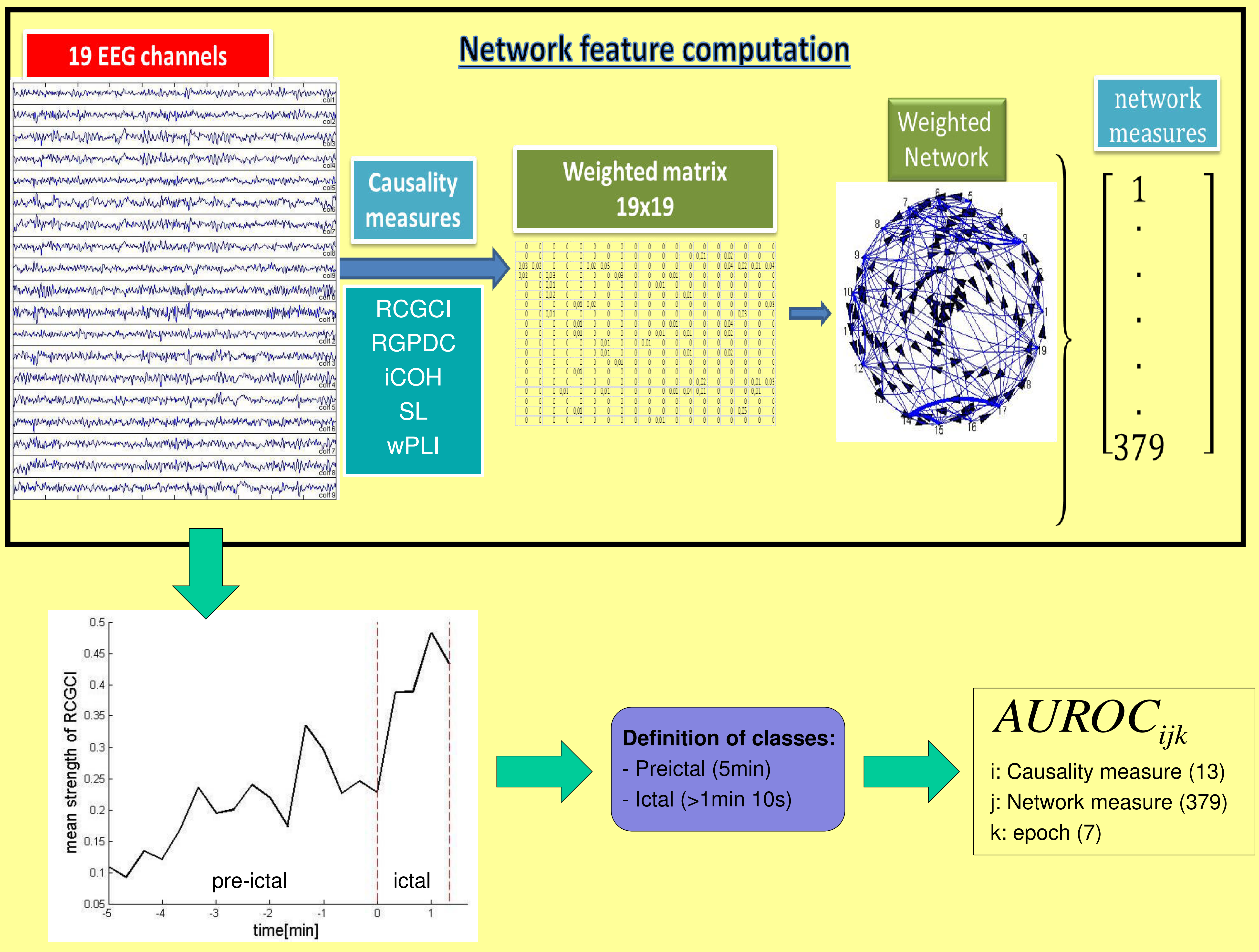

\section{Results}

Figure 1: Boxplots of network measure values before and during the seizure of one epoch. (a) Global clustering coefficient, (b) mean strength. Both network measures are computed on networks generated by the Granger causality index RCGCl

(a)

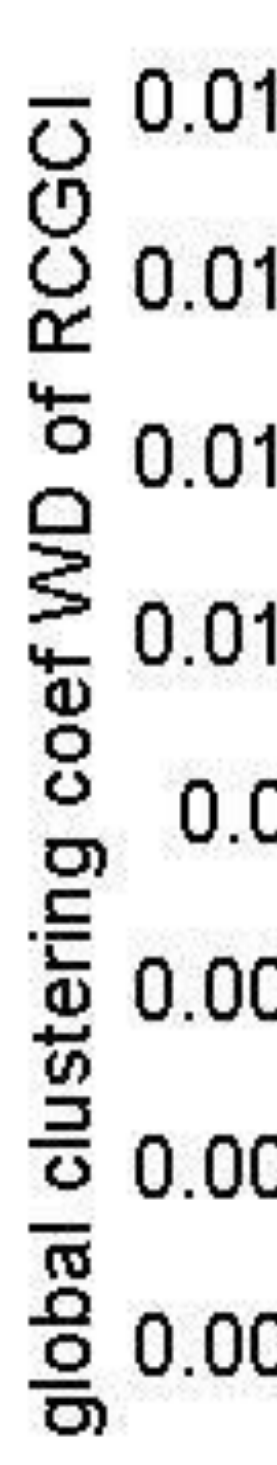

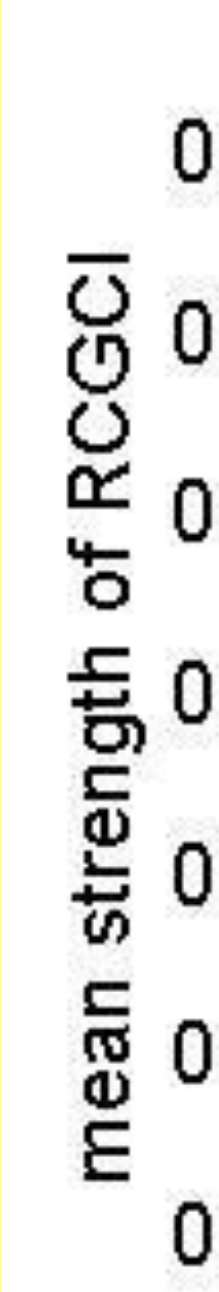

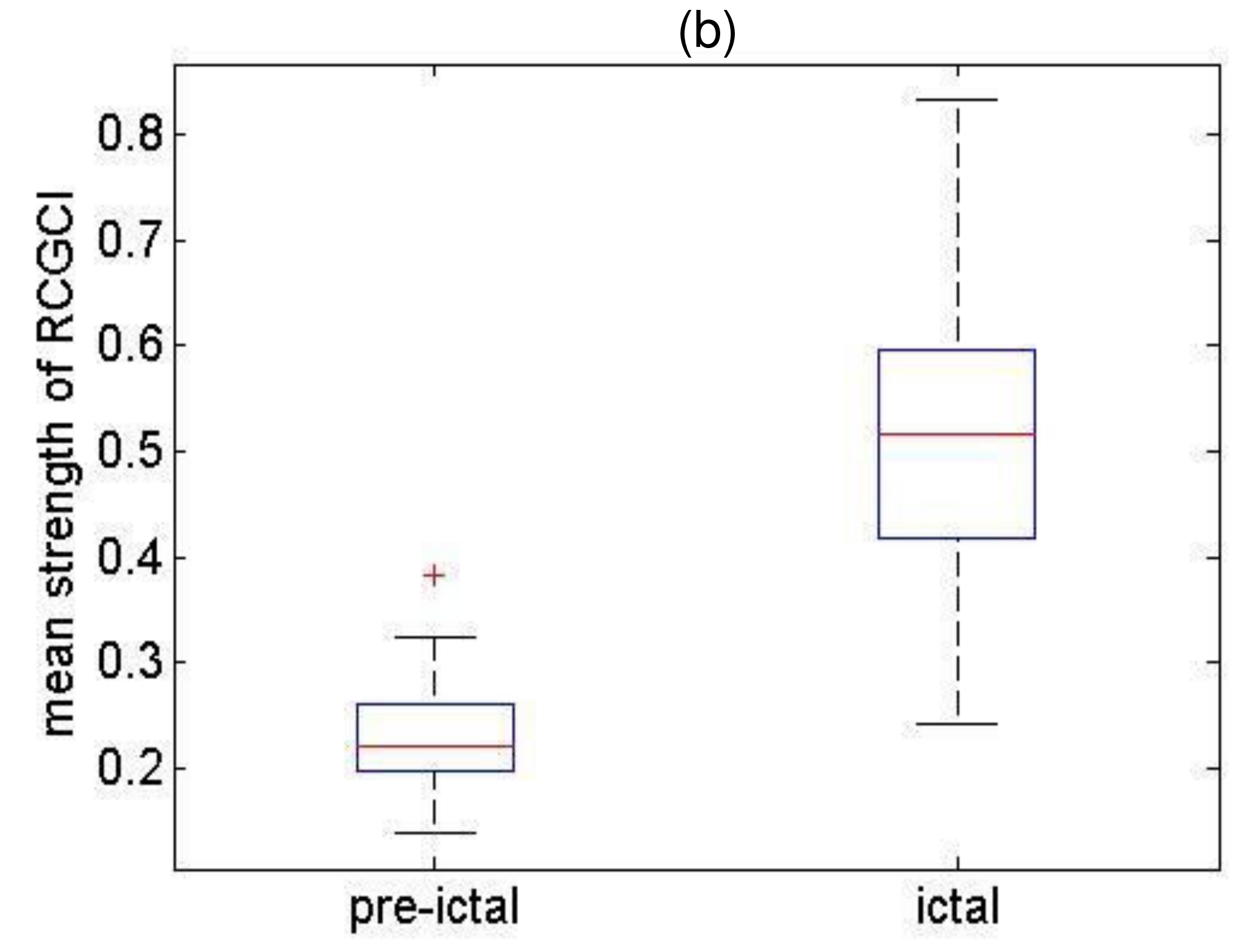

Figure 2: Network measure modul(5) on the pre-ictal and ictal period of all recordings. Network formulation with RGPDCa REC 1, AUROC: 0.98667
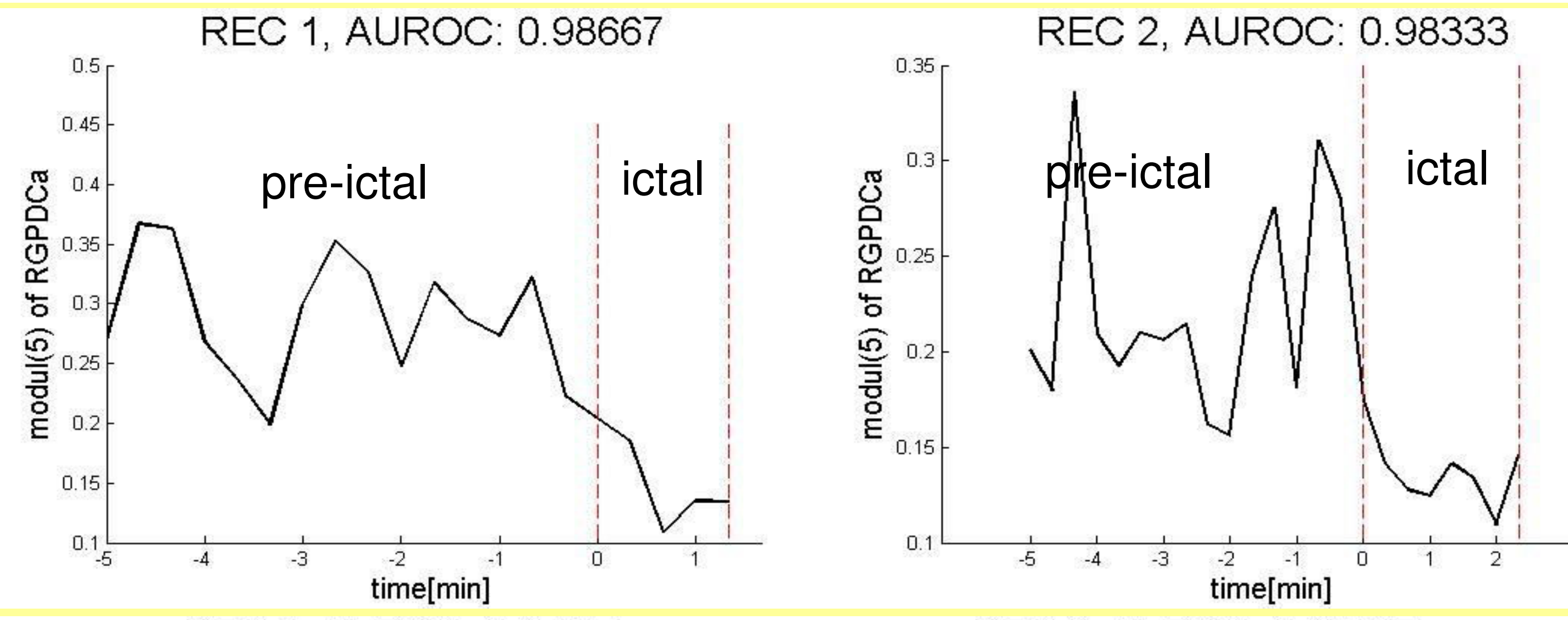

REC 5, AUROC: 0.81111
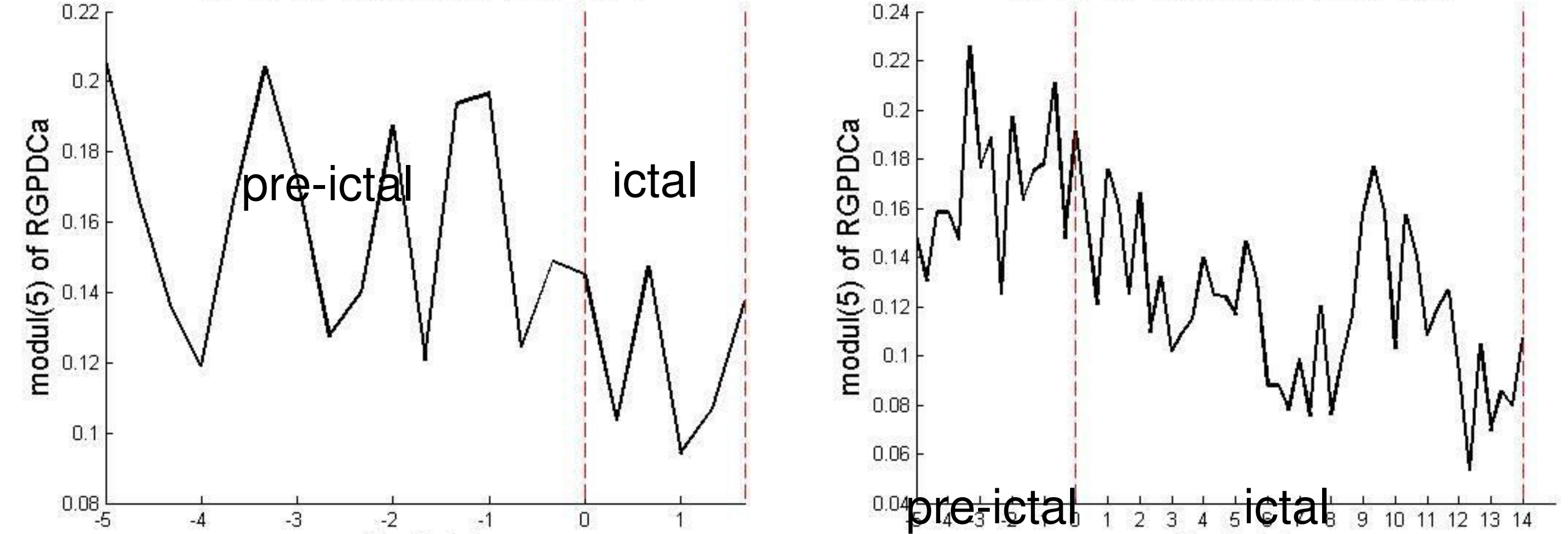

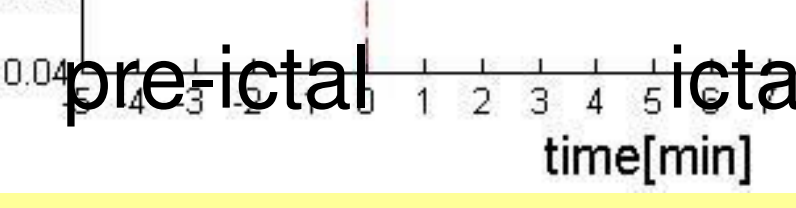

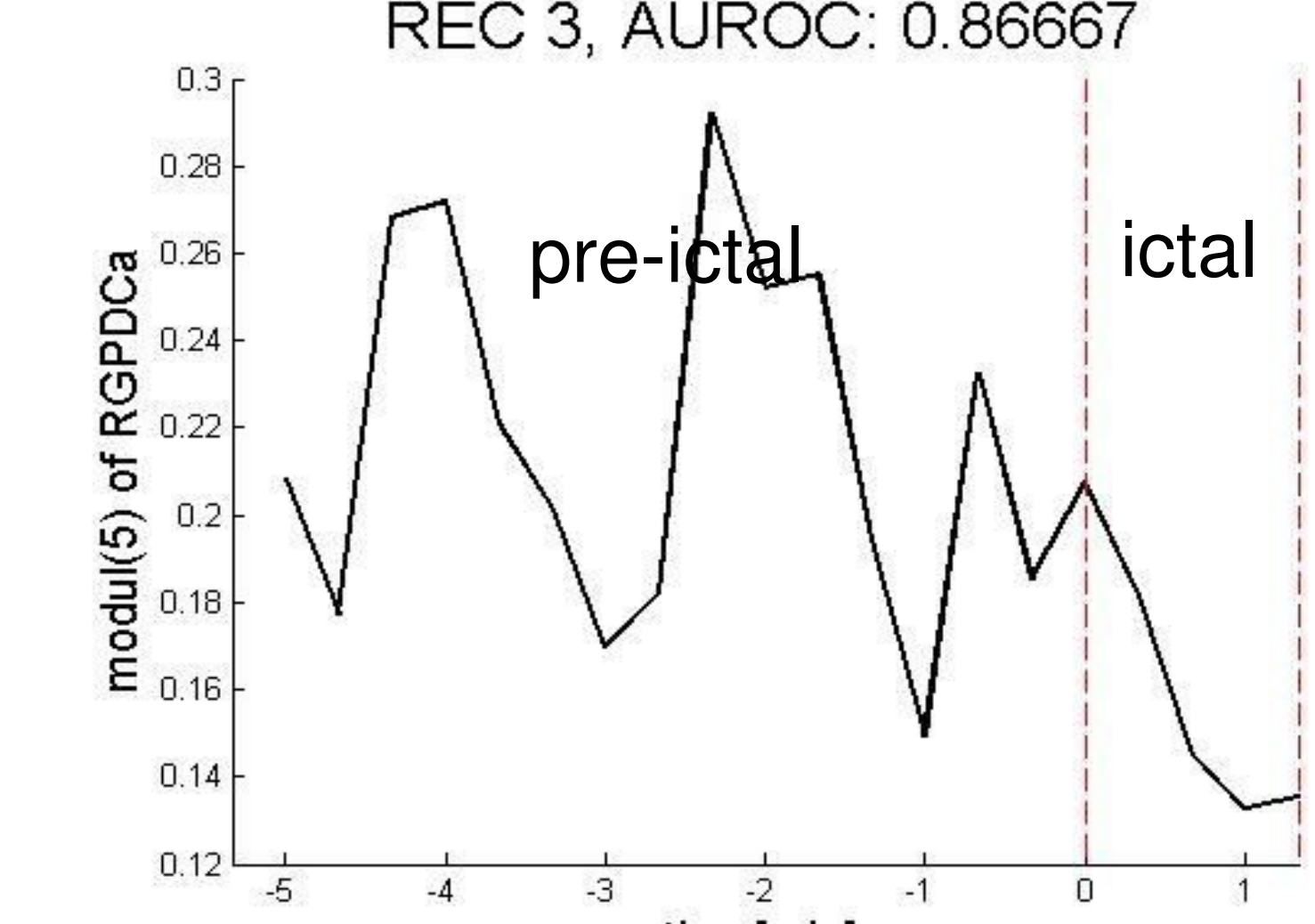

REC 7, AUROC: 0.79167
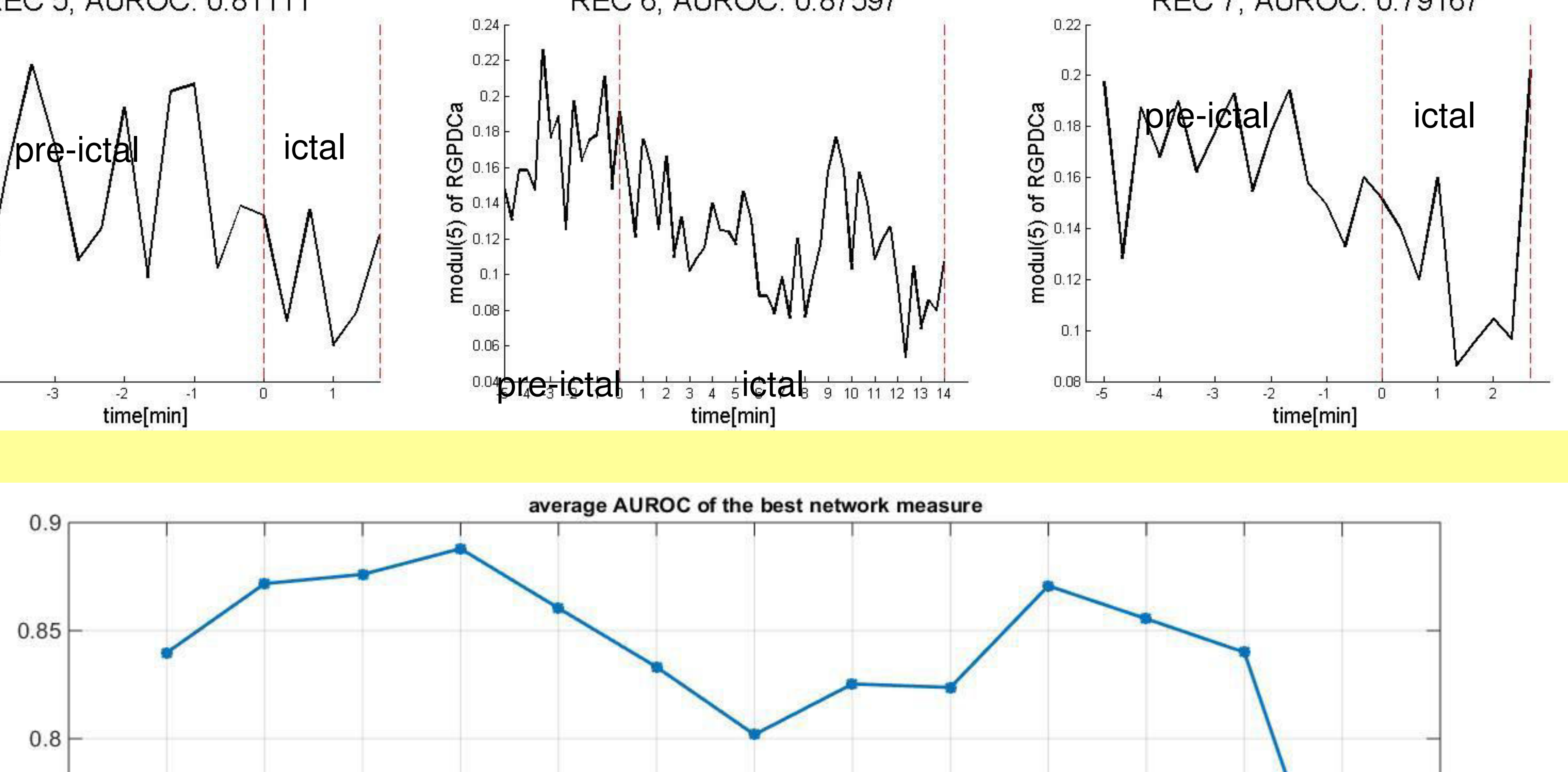

$\frac{1}{1} \frac{1}{1} \frac{1}{1} \frac{1}{1} \frac{1}{1} \frac{1}{1} \frac{1}{1} \frac{1}{1} \frac{1}{1}$

ation of Granger causality measures. Each causality measure is assigned the average A (over 7 epochs) of the network measure with the highest rank for this causality measure.
REC 6, AUROC: 0.87597

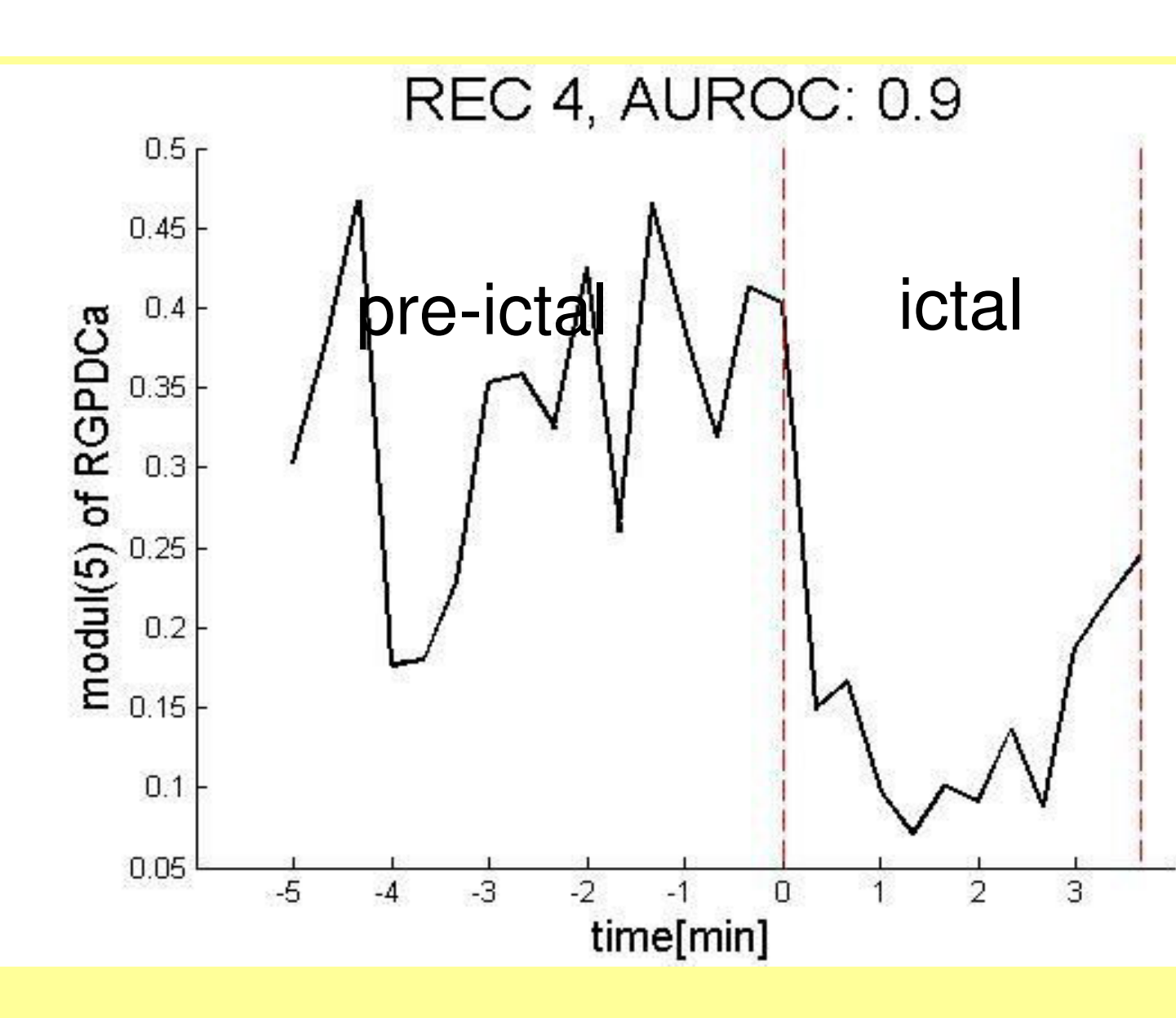

\begin{tabular}{|c|c|c|}
\hline $\begin{array}{l}\text { Causality } \\
\text { index }\end{array}$ & Network measure & $\begin{array}{c}\text { Avg } \\
\text { AUROC }\end{array}$ \\
\hline RGPDCa & modul(5) & 0.887 \\
\hline iCOHb & str in channel P3 & 0.870 \\
\hline RGPDCd & modul(3) & 0.861 \\
\hline iCOHg & str channel F10 & 0.855 \\
\hline iCOHg & clustering coef WD F10 & 0.853 \\
\hline iCOHb & clustering coef WD Cz & 0.852 \\
\hline RGPDCd & mean in strength & 0.851 \\
\hline RGPDCd & mean out strength & 0.851 \\
\hline RGPDCd & mean strength & 0.851 \\
\hline $\begin{array}{l}\text { RGPDCd } \\
\text { Table } 1: t \\
\text { combined } \\
\text { averag }\end{array}$ & $\begin{array}{l}\text { global clustering coef WD } \\
\text { the top } 10 \text { features (causa } \\
\text { with network measure) in } \\
\text { ge AUROC value over } 7 \text { e }\end{array}$ & $\begin{array}{l}0.848 \\
\text { lity index } \\
\text { terms of } \\
\text { oochs }\end{array}$ \\
\hline
\end{tabular}

References

[1] A. Papana, C, Kyrtsou,

Entropy, 15,2635-2661,(2013)

[2] E. Siggiridou and D.

Kunger causalty in multivate

[3] D. Kugiumtzis, V. K. Kimiskidis, "Direct causal networks for the study of 